\title{
Treatment of Multiple Common Warts by Intralesional Immunotherapy: Review Article
}

Aya Elsayed Elaraby*, Ahmad Abd Elgawad Nofal, Basma Magdy Elkholy

Dermatology, Venereology and Andrology Department, Faculty of Medicine, Zagazig University, Egypt

*Corresponding Author: Aya Elsayed Elaraby, E-Mail: ayaelsayed1319@gmail.com

\begin{abstract}
Background: Human papillomavirus (HPV) is the most prevalent cause of warts on the skin and mucous membranes of the human body. With the aid of a variety of destructive and immunotherapeutic methods, wart therapy remains a major difficulty. Antigen immunotherapy by intralesional technique uses the immune system's ability to recognize viral, bacterial, and fungal antigens that elicit a delayed-type hypersensitivity reaction, not only to the antigen but also to the wart virus, which in turn boosts the immune system's ability to recognize and remove HPV. All lesions on the body, not just the injected one, could be destroyed by this boosted immune response. Different antigens, including Candida and pure protein derivative (PPD), have been shown to be useful in the treatment of various wart forms. Objective: Intralesional immunotherapy for numerous common warts is to be evaluated for its effectiveness. Conclusion: There has been substantial research into the use of intralesional vaccinations and organic antigens to treat warts, with good results of effectiveness.
\end{abstract}

Keywords: Intralesional immunotherapy, Multiple common warts.

\section{INTRODUCTION}

The human papillomavirus (HPV) causes warts, which are frequent, benign proliferations of the epidermis. However, despite the fact that they can be seen allover, it is more typical to observe them on face, genitalia, feet and hands. Around seven percent to ten percent of patients seeking medical advice in dermatology departments worldwide are affected by warts. The most prevalent sexually transmitted disease is genital warts. Infection with HPV causes the formation of verrucae, which are typically 1 - to 20 -mm-diameter epithelial proliferations on mucus membranes and the skin ${ }^{(\mathbf{1})}$.

Medically, warts are frequent, especially among white people. 11.7 percent of the world's population is expected to have HPV, Eastern Europe (21.4 percent), Eastern Africa (33.6 percent), and the Caribbean (35.4 percent).
Children are more likely to have them. Sexually active women are estimated to have 12 percent of genital warts (2). There are more than 150 different varieties of this nonenveloped DNA virus. Sexual contact can spread some HPV strains. Towels and washcloths, for example, are common objects that most diseases are conveyed through skin-touch. Breaks in the skin, like a hangnail or a scrape, are the most common ways the virus spreads to new cases. Warts on the fingertips and in the vicinity of the nails can also spread as a result of nail biting. HPV causes warts in some people, however not all people who come into touch with the virus develop warts ${ }^{(3)}$.

The aim of this review of intralesional immunotherapy for numerous common warts is to be evaluated for its effectiveness.

\section{Types of warts (Figure 1):}

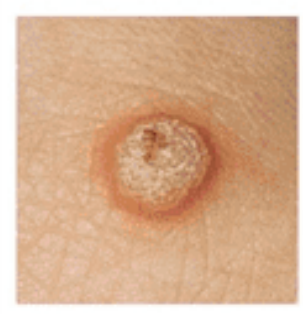

common wart

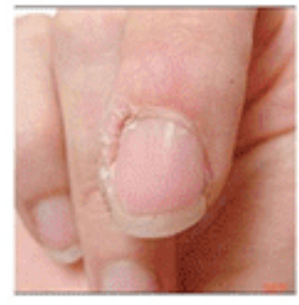

periungual wart

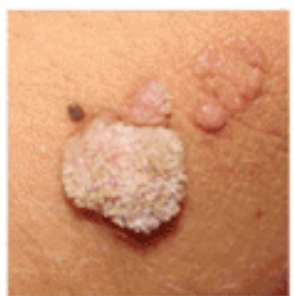

filiform wart

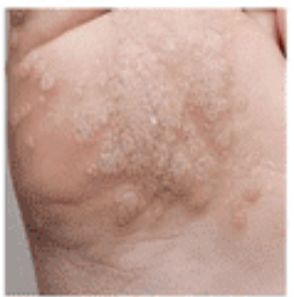

mosaic wart

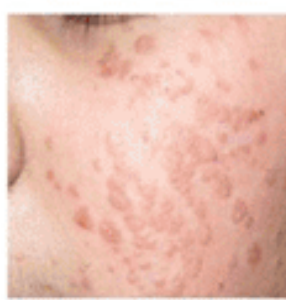

flat wart

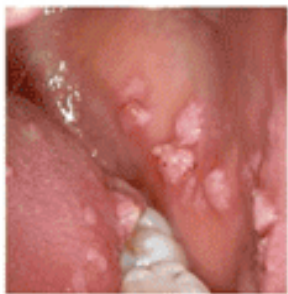

oral wart

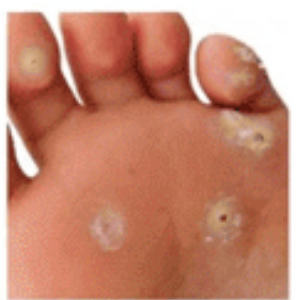

plantar wart

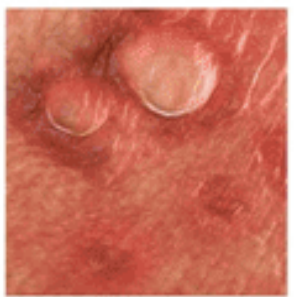

genital wart

Figure (1): Different types of warts ${ }^{(4)}$. 


\section{Intralesional Immunotherapy:}

According to the definition, immune-suppressing and immune-stimulating chemicals are used in immunotherapy to aid the body's fight against infection and cancer. Immunotherapies that target only a subset of the immune system are known as cellular immunotherapy. Cytokines, vaccinations, and certain monoclonal antibodies are examples of immunotherapy. It could be an immunotherapy that induces or enhances immunity, called activation immunotherapy (used for tumors and infection), or an immunotherapy that suppresses the immune system (used in autoimmune diseases). Using immunotherapy to treat cancer has been around for a long time, but it has just found a place in the treatment of infections ${ }^{(5)}$.

Traditional methods of treatment can only be used locally and do not have a systemic effect. As a result, patients who have several distant lesions find them inconvenient. Interferons and contact sensitizers have been used to boost the host's immune response in a variety of ways. Additionally, intralesional injections of vaccinations and organic antigens have had some success. Among the antigens investigated are Candida albicans, measles, mumps, and rubella, (MMR); purified protein derivative (PPD), Mycobacterium vaccine, Bacillus Calmette-Guerin (BCG), and Trichophyton antigens ${ }^{(6)}$.

Immunotherapy for warts has no well-defined criteria or consensus on when it should be used. The most recent findings include recurrent, recalcitrant, extensive, periungual and palmoplanter warts, which are difficult to treat. Using this method, the body's T-cells are activated in a systemic manner. Interleukin-2 and interferongamma, which are mostly generated by Th1 cells, are among the cytokines that respond most strongly to injection. Additionally, intralesional injection may concentrate the local immune response, as well as elicit an adequate immunological response in immunocompetent patients through the trauma of injection. Additional advantages of immunotherapy include its lower cost than alternative treatment options ${ }^{(7,8)}$.

Interlesionally, immunogenic protein antigens have been employed to treat warts. Immunogenic qualities of each antigen are unique to each individual patient, and the same antigen might produce varied reactions depending on the patient's particular characteristics. The host response to an antigen may be weak or nonexistent in some situations. Intradermal injection and measurement of induration and erythema can be used to screen for antigen sensitivity prior to intralesional therapy. Pre-sensitization of the patient can also be achieved by this technique. There is evidence that intralesional immunotherapy can be effective in the treatment of warts, but individual outcomes are highly diverse ${ }^{(6)}$.

The most commonly reported side events are brief flu-like symptoms and erythema and edema of the injection site, both of which are well tolerated. Different antigens have been employed with a variety of dosing methods in an attempt to discover the regimen that gives the best results ${ }^{(9)}$.

\section{1) Candida albicans:}

Intralesional Candida albicans antigen has been explored by several groups for the treatment of warts. In research including 34 patients with intractable verruca vulgaris, Majid and Imran ${ }^{(10)}$ utilized $0.1 \mathrm{~mL}$ of Candida antigen intralesionally every three weeks for a total of three sessions. As a whole, $56 \%$ of those who had treatment for warts saw their symptoms disappear, while $6 \%$ showed some improvement. Within six months, none of the patients experienced recurrence.

In an effort to boost immunogenicity, doses greater than $0.1 \mathrm{~mL}$ have been employed with inconsistent outcomes. In order to treat 11 patients with non-genital, non-facial warts, Kim et al. ${ }^{\left({ }^{11}\right)}$ injected $0.3 \mathrm{~mL}$ of Candida antigen every three weeks. A total of four treatments were given to each patient. 82 percent of the nine patients experienced complete remission, and one ( 9 percent) had a partial response with no deterioration in symptoms. Using higher doses is definitely recommended to obtain a greater immunogenic response based on these findings. Twenty patients with warts were involved in the study by Attwa et al. ${ }^{(12)}$, who injected Candida antigen. There is no limit to how many sessions you can have until the lesions are completely cleared or until you reach the maximum number of sessions allowed. In only four cases, lesions completely disappeared; in five, only modest to moderate responses were observed.

\section{2) Mumps or Candida:}

Intradermal reactions to two separate antigens were tested for the first time by Johnson et al. ${ }^{(13)}$ to decide which antigen should be used intralesionally in each patient. Antigen injections for verruca vulgaris were administered intradermally in two separate forearms: $0.1 \mathrm{mg}$ of mumps antigen and $0.1 \mathrm{mg}$ of Candida antigen. After 48-72 hours, they measured the reactivity to each antigen, which necessitated at least a $5 \mathrm{~mm}$ induration with erythema to be considered positive. Intradermal injections were only used in the trial if a patient was responsive. Intralesional therapy was performed with the antigen that elicited the strongest intradermal reaction.

\section{3) Mumps, measles, and rubella:}

Immune responses to the host have been induced by administering the MMR vaccine intravenously as well. The MMR vaccine or saline was given to 46 patients at random. Two-week intervals were employed to administer a normal injection dose of $0.5 \mathrm{~mL}$. MMR vaccine injections resulted in 18 (75\%) full responses and four (17\%) partial responses in the 24 patients who received them. Patients with flu-like symptoms accounted for $29 \%$ of all cases ${ }^{(\mathbf{1 4})}$.

Nofal and Fouda ${ }^{(9)}$ conducted a new study comparing the intralesional use of candida and the MMR vaccine in the treatment of warts. For this study, 68 adolescent patients with multiple common and plantar warts were divided into two groups, each with 34 participants. The MMR vaccine was administered intralesionally to the first group, whereas the Candida antigen was administered intralesionally to the second. The largest wart was injected 
every two weeks until it was completely gone or for a maximum of five treatments. Candida antigen group had a 73.5 percent success rate compared to the MMR group's 66.7 percent, but the difference was insignificant. The Candida antigen group had a greater clearance rate of common warts than the MMR group, while the MMR group had a higher clearance rate of plantar warts than the Candida group. Both groups experienced brief and welltolerated side effects. The 6-month follow-up period found no recurrence ${ }^{(9)}$.

\section{4) Mycobacterium:}

Warts can also be treated using mycobacterial antigens, which have been used in the past. PPD, Mycobacterium w vaccine (MWV), and Bacillus Calmette-Guerin (BCG) have been explored for this purpose ${ }^{(\boldsymbol{6})}$.

A) Purified protein derivative: When it comes to detecting tuberculosis infections, PPD is the most widely utilized method in the world ${ }^{(15)}$. Intradermal injections of tuberculin antigens are followed by observation of induration at the injection site. PPD intralesionally injected into warts has been the subject of three clinical investigations. 55 individuals with previously untreated or recalcitrant warts were treated by Saoji $\boldsymbol{e t ~ a l} .{ }^{(16)}$ with 0.05 $\mathrm{mL}$ of PPD per lesion. More than seventy percent of patients were cured after four two-week periods of treatment. Less than 1 in 10 patients developed new lesions six months following the initial procedure. One patient suffered a fever, another developed transitory eyelid edema following injection into the eyebrow, and a third developed an eczematous reaction to the treatment. Tuberculin PPD antigen injection intralesionally compared to MMR antigen injection was studied by Mohammed et $\boldsymbol{~ a l} .{ }^{(17)}$ for safety and efficacy in treating multiple warts. There were 90 participants in the study, all of whom were categorized into three categories (A, B, and $\mathrm{C})$. There were thirty patients in each group. Intralesional injections of PPD were administered to Group (A) individuals. Intralesional injections of MMR antigen were given to those in group (B), while intralesional injections of saline were given to those in group (C). 60 percent of patients in group A cleared their warts completely, compared to 80 percent of patients in group B who had cleared their warts completely. Eighteen of the patients in group A (60 percent) and twelve of the patients in group B (40 percent) no longer had any distal warts. Pain, erythema, and minor induration were observed seldom in both groups.

B) Mycobacterium $w$ vaccine: To cure warts, researchers have used MWV, which comprises particles of a fastgrowing atypical mycobacterium with immunogenic potential ${ }^{(\mathbf{1 8})}$. In an open pilot trial including nine patients, Gupta et al. ${ }^{(19)}$ investigated the efficacy of MWV in the treatment of anogenital warts. After an average of 6.3 treatments, eight patients (or $89 \%$ of the cohort) were fully recovered. Edema at the injection site was the most often reported side effect. However, granulomatous balanitis and herpes zoster were also documented in patients. An initial pilot trial including 40 patients with an average of
9.7 weekly injections was undertaken by Meena et al. ${ }^{(20)}$. Eighty-two percent of the patients (33) got complete resolution, whereas four individuals had partial response (10 percent). Only $15 \%$ of patients experienced edema, while $5 \%$ of patients experienced fevers.

C) Bacillus Calmette-Guerin: Three cases of intralesional BCG's success have also been documented. Condyloma acuminata of the penis was resistant to previous medical treatment in two cases reported by Gupta ${ }^{(21)}$. Intralesional injections of BCG were administered to each subject. No recurrence of the lesions was observed in both patients at six-month follow-up visits following treatment. After three or five treatments with $0.1 \mathrm{~mL}$ BCG, Kumar and Das ${ }^{(22)}$ reported full eradication of stubborn non-genital warts. In both cases, the therapy was accompanied with short-lived flu-like symptoms.

\section{5) Trichophyton skin antigen:}

An allergenic extract of Trichophyton species has been made by adding extracting solution. More than $62 \%$ of patients who received intralesional Trichophyton injections ( $0.3 \mathrm{ml}$ every 3 weeks, maximum of 5 sittings) reacted, and the response was not significantly different from that of injections of mumps antigen and Candida antigen with interferon- $\alpha(\text { IFN } \alpha)^{(9)}$. Plenty of research has been done with trichophyton alone. One study found a 71 percent response rate when combined with Candida and mumps antigens, which was much higher than the response rate of separate antigens ${ }^{(9)}$.

\section{6) Interferons:}

An in-vivo role is played by tiny proteins called interferons, which are produced by the body. Immunomodulatory, antiviral, and antiproliferative characteristics of IFN $\alpha-2 B$ have been employed as therapeutic agents. When it comes to treating warts, there are a number of conflicting findings. Genital warts respond significantly better to therapy with topical interferons than placebo, according to a systematic evaluation of 12 randomized controlled studies including 1445 individuals (44.4 percent vs 16.1 percent). A substantial difference in response between systemic interferons and a placebo, on the other hand, was not observed (27.4 percent vs 26.4 percent) ${ }^{(\mathbf{1 7})}$.

\section{7) Human papilloma virus vaccines:}

As genital warts reduce in Denmark, the quadrivalent HPV vaccination containing the L1 protein of HPV types $6,11,16$, and 18 has been widely used. The HPV vaccine reduced the likelihood of warts over time. After receiving three doses, patients were completely protected from genital warts, whereas those who received two doses had a further decrease in genital wart incidence as the interval between them increased ${ }^{(23)}$. The drop of genital warts in the UK and Australia was similar ${ }^{(24)}$.

Immunocompetent and immunocompromised persons alike have reported success with HPV vaccination treatment for cutaneous warts. HPV-related illnesses such as warts are predicted to drop even more with the creation 
of nonavalent HPV vaccines against HPV types 6, 11, 16, $18,31,33,45,52$ and $58^{(25)}$.

\section{8) Vitamin D:}

Some research has shown that vitamin D supplementation can help treat warts. The use of IL vitamin D for the treatment of plantar warts was initially reported by Aktas et al. ${ }^{(26)}$. A maximum of two injections of vitamin D3 at monthly intervals $(0.2,7.5 \mathrm{mg} / \mathrm{mL})$ resulted in the total clearance of 80 percent of the individuals with warts. The following studies, by Kavya et al. ${ }^{(27)}$ and Raghukumar et al. (28) employed IL vitamin D3 and reported full clearance in $78.6 \%$ of patients and $90 \%$ of patients, respectively. Vitamin D3 of higher concentrations (0.2$0.5 \mathrm{~mL}$ of $15 \mathrm{mg} / \mathrm{mL}$ ) were injected into more than one wart at a time for four therapy sessions.

\section{CONCLUSION}

There has been substantial research into the use of intralesional vaccinations and organic antigens to treat warts, with some good results of their effectiveness.

\section{Financial support and sponsorship: Nil. Conflict of interest: Nil.}

\section{REFERENCES}

1. El-Mohamady A, Mearag I, El-Khalawany M et al. (2014): Pulsed dye laser versus Nd:YAG laser in the treatment of plantar warts: A comparative study. Lasers Med Sci., 29(3):1111-1116.

2. Soheili M, Keyvani H, Soheili M et al. (2021): Human papilloma virus: A review study of epidemiology, carcinogenesis, diagnostic methods, and treatment of all HPVrelated cancers. Medical Journal of the Islamic Republic of Iran, 35(1): 481-496.

3. Kermott C, Plummer M, Vignat J et al. (2017): Worldwide burden of cancer attributable to HPV by site, country and HPV type. International journal of cancer, 141(4): 664-670.

4. Ghadgepatil S, Gupta S, Sharma Y (2016): Clinicoepidemiological study of different types of warts. Dermatology Research and Practice, 16: 1-6.

5. Thappa D, Chiramel M (2016): Evolving role of immunotherapy in the treatment of refractory warts. Indian Dermatology Online Journal, 7(5): 364-69.

6. Aldahan A, Mlacker S, Shah V et al. (2016): Efficacy of intralesional immunotherapy for the treatment of warts: A review of the literature. Dermatologic Therapy, 29(3): 197-207.

7. Nofal A, Nofal E, Yosef A et al. (2015): Treatment of recalcitrant warts with intralesional measles, mumps, and rubella vaccine: a promising approach. International Journal of Dermatology, 54(6): 667-671.

8. Sefcik R, Burkhart C (2017): Wart immunotherapies: A short review. The Open Dermatology Journal, 11(1): 1-5.

9. Nofal A, Fouda I (2020): Switching between intralesional antigens: A promising therapeutic approach for recalcitrant warts. JAAD Case Reports, 6(10): 1032-36.

10. Majid I, Imran S (2013): Immunotherapy with intralesional Candida albicans antigen in resistant or recurrent warts: A study. Indian Journal of Dermatology, 58(5): 360-66.

11. Kim K, Horn T, Pharis J et al. (2010): Phase 1 clinical trial of intralesional injection of Candida antigen for the treatment of warts. Archives of Dermatology, 146(12): 1431-1433.
12. Attwa E, Elawady R, Salah E (2020): 'Cryo-immunotherapy' is superior to intralesional Candida antigen monotherapy in the treatment of multiple common warts. Journal of Dermatological Treatment, 20: 1-8.

13. Johnson S, Roberson P, Horn $T$ (2001): Intralesional injection of mumps or Candida skin test antigens: a novel immunotherapy for warts. Archives of Dermatology, 137(4): 451-455.

14. Zamanian A, Mobasher P, Jazi G (2014): Efficacy of intralesional injection of mumps-measles-rubella vaccine in patients with wart. Advanced Biomedical Research, 3: 107-111.

15. Yang H, Kruh-Garcia N, Dobos K (2012): Purified protein derivatives of tuberculin-past, present, and future. FEMS Immunology \& Medical Microbiology, 66(3): 273-280.

16. Saoji V, Lade N, Gadegone $R$ et al. (2016): Immunotherapy using purified protein derivative in the treatment of warts: An open uncontrolled trial. Indian Journal of Dermatology, Venereology and Leprology, 82(1): 1-4.

17. Mohammed Y, Ibrahim H, Elbarbary M et al. (2021): Comparative study of intralesional tuberculin protein purified derivative (PPD) and intralesional measles, mumps, rubella (MMR) vaccine for multiple resistant warts. Journal of Cosmetic Dermatology, 20(3): 868-874.

18. Talwar G, Sarathchandra K, Walia $R$ et al. (2005): Immunoprophylactic effects of the anti-leprosy Mw vaccine in household contacts of leprosy patients: clinical field trials with a follow up of 8-10 years. Lepr Rev., 76: 127-143.

19. Gupta S, Malhotra A, Verma K et al. (2008): Intralesional immunotherapy with killed Mycobacterium w vaccine for the treatment of ano-genital warts: an open label pilot study. Journal of the European Academy of Dermatology and Venereology, 22(9): 1089-1093.

20. Meena J, Malhotra A, Mathur D et al. (2013): Intralesional immunotherapy with Mycobacterium $w$ vaccine in patients with multiple cutaneous warts: Uncontrolled open study. JAMA Dermatology, 149(2): 237-239.

21. Gupta S (2016): Single dose intralesional immunotherapy with BCG of medically resistant condylomata acuminata of the penis: report of two cases. Int J Dermatol., 55(5): 583-6.

22. Kumar P, Das A (2014): Excellent response to intralesional bacillus Calmette-Guérin vaccine in a recalcitrant periungual wart. Journal of Cutaneous and Aesthetic Surgery, 7(4): 23438.

23. Blomberg M, Dehlendorff C, Sand C et al. (2015): Doserelated differences in effectiveness of human papillomavirus vaccination against genital warts: A nationwide study of 550 000 young girls. Clinical Infectious Diseases, 61(5): 676-682.

24. Chow E, Read T, Wigan R et al. (2015): Ongoing decline in genital warts among young heterosexuals 7 years after the Australian human papillomavirus (HPV) vaccination programme. Sexually Transmitted Infections, 91(3): 214-219.

25. Pham C, Juhasz M, Sung C et al. (2020): The human papillomavirus vaccine as a treatment for human papillomavirus-related dysplastic and neoplastic conditions: A literature review. Journal of the American Academy of Dermatology, 82(1): 202-212.

26. Aktas H, Ergin C, Demir B et al. (2016): Intralesional vitamin D injection may be effective treatment option for warts. Journal of Cutaneous Medicine and Surgery, 20: 118-122.

27. Kavya M, Shashikumar B, Harish M et al. (2017): Safety and efficacy of intralesional vitamin D3 in cutaneous warts: An open uncontrolled trial. Journal of Cutaneous and Aesthetic Surgery, 10: 90-94.

28. Raghukumar S, Ravikumar B, Vinay K et al. (2017): Intralesional vitamin D3 injection in treatment of recalcitrant warts: A novel proposition. Journal of Cutaneous Medicine and Surgery, 21: 320-324. 